\title{
Exploring the Barkas effect with keV-electron scattering
}

\author{
P. L. Grande ${ }^{*}$ \\ Department of Electronic Materials Engineering, Research School of Physics and Engineering, The Australian National University, \\ Canberra, Australia 0200 and Instituto de Fisica da Universidade Federal do Rio Grande do Sul, Avenida Bento Goncalves 9500, 91501-970, \\ Porto Alegre, RS, Brazil \\ M. Vos \\ Atomic and Molecular Physics Laboratories, Research School of Physics and Engineering, The Australian National University, \\ Canberra, Australia 0200 \\ (Received 25 September 2013; published 1 November 2013)
}

\begin{abstract}
The energy loss of fast ions at close collision is mainly due to electron-ion collisions. The electrons are approximately stationary and they collide with a fast-moving ion. Here we study the same collision experimentally, in a reference system where the ions (or atoms) are stationary and interacting with keV electrons. Scattering cross sections under these conditions deviate from Rutherford, and we link these deviations, at higher energies, to the $Z^{3}$ contributions to the electronic stopping and the related Barkas effect and, at lower energies, also to quantum interference. The present measurements are well described by partial-wave calculations of the elastic cross section of electrons scattering from atoms. Encouraged by this agreement we use these calculations to estimate the Barkas factor for all elements and many energies. A universal curve for the Barkas factor due to close collisions is obtained for neutral projectiles and similar curves with smaller magnitude are found for ions.
\end{abstract}

DOI: 10.1103/PhysRevA.88.052901

PACS number(s): $34.50 . \mathrm{Bw}, 34.80 . \mathrm{Bm}, 34.50 . \mathrm{Fa}$

\section{INTRODUCTION}

The slowing down of energetic particles in matter has been an active area of research since the early days of quantum physics. Bethe formulated a very successful theory describing the energy loss as the consequence of collisions between the fast-moving ion and nearly stationary electrons. In this approach, based on perturbation theory, the energy loss is proportional to the square of the charge of the projectile, $Z^{2}[1]$, and therefore the energy loss is independent of the sign of the charge. Careful measurements by Andersen and coworkers [2] indicated that this is not quite true, there were small, but significant contributions to the stopping proportional to $Z^{3}$ and $Z^{4}$. The term proportional to $Z^{3}$ is intriguing, as it suggest that the stopping power of a particle may differ from that of its antiparticle. Indeed this was later confirmed by experiments comparing the stopping of protons and antiprotons [3-5]

Such effects that depend on the sign of the charge of a particle have always fascinated scientists since they are a stringent test of the understanding of the underlying physics. In general it is not an easy task to perform experiments with negatively and positively charged projectiles (or targets) since it involves the production of antimatter. When possible, these charge-sign-dependent effects provide a key test of our understanding. The first indications of such a dependence came from the work of Barkas and coworkers [6], who observed different ranges for positive and negative pions in matter. Initially this observation was attributed to a difference in the pion masses, but latter it was demonstrated that it is due to higher-order contributions to the energy loss rate or stopping power $[7,8]$. From then on a difference that depends on the sign of the charge is referred to as a Barkas effect. Its origin was initially ascribed to polarization, as a medium reacts differently

\footnotetext{
*Corresponding author: grande@if.ufrgs.br
}

to positively and negatively charged particles [9]. In this picture positively (negatively) charged particles attract (repel) the electron cloud giving rise, in a second-order process, to an enhancement (a depletion) of the electronic density around the ion path which in turn increases (decreases) the energy loss. Several years later Lindhard [10] suggested that projectile screening also leads to different energy losses depending on the sign of the charge of the projectile. This means that for close collisions (when the energy loss is dominated by momentum transfer of individual electrons from the medium to the projectile), the sign of the charge of the projectile is important. There are two reasons for the screening of the projectile nuclear charge. One is the rearranging of the valence electrons. This results in a screening length determined by the plasmon frequency (i.e., the electron density) of the target material. A second screening mechanism exists for partially ionized ions, also referred to as dressed ions. Here the remaining core electrons screen the nuclear charge and, as explained later, also cause a Barkas effect which is largely independent of the target electronic structure. We are concerned here mainly with the latter type of screening.

Apart from a few exceptions [11-16] most of the theoretical work published after the paper of Lindhard [10] has treated the Barkas effect as a consequence of close collisions [17-28]. The Barkas effect at close collision not only affects the stopping power but also manifests itself in other physical areas, but the corresponding interconnections are less known. One such example is the binary-encounter-peak enhancement for partially stripped ions [29-31]. Another example is keVelectron scattering [32-35]. The latter experiment describes the scattering in the reference frame where the ion (or atom) is at rest. Such an experiment is relevant for stopping theory, as the momentum transfer rate (and as a consequence the stopping power) is determined by the elastic scattering cross section of the electrons in the reference frame where the screened ion is at rest [17]. These scattering experiments are 
used nowadays to characterize thin films and small molecules $[35,36]$. They are the electron analog of the well-established (ion) Rutherford backscattering spectrometry (RBS) [37] and we refer to it as ERBS (electron RBS). The element selectivity is based on the recoil energy transferred by the $\mathrm{keV}$ electron under backscattering conditions. As in RBS, the elemental quantification is accomplished by the differential elastic cross section $d \sigma / d \Omega$, which is determined by the atomic potential. Conversely if the atomic composition is known we can compare the ratio of the cross section of two elements to the calculated one. It turns out that the cross section does not scale exactly as $Z^{2}$ and we can use these experiments thus to elucidate higher-order contributions to the cross section.

In this work we explore the similarities of the Barkas effect for stopping power with the screening effect found in keV-electron scattering [33] to quantify the Barkas effect for near central collisions (impact parameters close to zero). In these experiments we use targets consisting of two elements with a well-defined stoichiometry. The elastic peak of both elements are resolved due to the recoil effect and an enhanced intensity of the heavy element is observed, which is interpreted as a sign of the Barkas effect. The results from highenergy electron-scattering experiments of oxides, water (ice or gas), and methane (gas) are used to validate the theoretical calculations from Salvat and coworkers [38,39], which were used here to calculate the Barkas effect for many elements with different ionization degrees. The calculated results for neutral or strongly screened projectiles tend to collapse on a single universal curve at high velocities and small projectile charges. However, at high $\mathrm{Z}$ and low energies, in contrast to classical calculations from Ref. [25], the simple scaling breaks down due to quantum effects. These quantum effects are also demonstrated experimentally. We also show that, although there is a single universal curve representing the Barkas effect for neutral and bare ions, no simple scaling exists for other projectile charge states.

The remainder of the paper is organized as follows. In the next section we discuss the physics of ERBS. In Sec. III we show the connection between the Barkas effect at close collisions and high-energy electron scattering. Experimental results for the elastic cross section are shown in Sec. IV. In Sec. V we present the theoretical method for calculating the elastic cross section and the Barkas factor. Theoretical results are shown for neutral ions as well as for ions with different degrees of ionization. The results are summarized in Sec. VI. Unless otherwise indicated, atomic units are used throughout the paper.

\section{PHYSICS OF ERBS}

In these experiments we measure the energy of electrons (incoming energy $E_{0}$ ) backscattered from an atom (either part of a molecule in the gas phase or part of a solid), focusing on energies very close to the incoming energy. Here we separate the contribution of different elements at high momentum transfer $K$ (i.e., high incoming energy and large scattering angles). Under these conditions the impulse approximation applies [40] and the recoil energy $\left(E_{\text {rec }}\right)$, transferred to the scattering atom, can be determined assuming a collision between free particles (this is the impulse approximation):

$$
E_{\mathrm{rec}}=\frac{K^{2}}{2 M_{a}}=2 E_{0} \frac{m_{e}}{M_{a}}(1-\cos \theta),
$$

with $M_{a}$ and $m_{e}$ being the mass of the atom and the electrons, respectively, and $\theta$ being the scattering angle. The energy of the incoming electron is reduced by the recoil energy. The width of the elastic peaks is not just determined by the experimental resolution but also by Doppler broadening due to the momentum distribution (thermal vibration) of the scattering atom. This is well corroborated by experimental outcomes and the obtained spectra are very similar to neutron-Compton-scattering spectra at the same momentum transfer [41]. Details of the ERBS technique can be found elsewhere [33].

It is instructive to consider the ion stopping power $d E / d x$ in the context of Eq. (1). In the framework of the binary theory the stopping is due to fast ions scattering from electrons that are approximately at rest. In the reference frame where the electron is at rest the recoil energy is $M_{a} / m_{e}$ times larger than that given by Eq. (1). For an ion with velocity $v$ such that $v_{0} \ll v \ll c\left(v_{0}\right.$ is the Bohr velocity, $c$ is the speed of light) the stopping power is then given by [17]

$$
d E / d x=\int_{\Omega} N \frac{M_{a}}{m_{e}} E_{\mathrm{rec}}(\theta) \frac{d \sigma}{d \Omega} d \Omega=N m_{e} v^{2} \sigma_{\mathrm{tr}},
$$

with $N$ being the target electron density. The transport cross section $\sigma_{\text {tr }}$ is defined as

$$
\sigma_{\mathrm{tr}}\left(e^{-}\right)=\int_{\Omega}[1-\cos (\theta)] \frac{d \sigma}{d \Omega}\left(e^{-}\right) d \Omega,
$$

with $\frac{d \sigma}{d \Omega}\left(e^{-}\right)$being the differential cross section (DCS) for an electron scattering from atom $Z$.

\section{SCREENING EFFECT IN HIGH-ENERGY ELECTRON SCATTERING}

In ERBS the electrons do not scatter from a bare nucleus, and the effect of the atomic electrons on the scattering potential has to be taken into account. The naive view, generally encountered, is that the screening will reduce the scattering cross section as the nuclear charge is partly compensated by the core electrons, and hence the scattering cross section should be less.

In reality the screening results in an increase of the cross section, i.e., the elastic cross-section values are larger than the Rutherford cross section of the bare nucleus [33,36]. The explanation for this enhancement can be found in many places in the literature dealing with fast ions moving through matter $[10,24,30]$ and can be expressed, at least partly, in terms of classical mechanics. Large-angle deflections correspond to small impact parameters and the main deflection occurs very close to the nucleus. For a Coulomb potential the electrons are accelerated as they approach the nucleus, so their velocity near the nucleus is increased. In the case of a screened potential this prior acceleration is smaller. Therefore, for a screened nucleus the electrons will have a lower velocity near the nucleus. Since the cross section decreases with increasing velocity, the final effect of screening is an increase of the cross section relative to Rutherford. The distance of the closest approach also changes with screening but it can be shown that this effect 
is less important than the change of the local velocity due to the screening [24]. This mechanism was initially proposed by Lindhard [10], who used the expansion of the Yukawa screening potential at small distances to derive an energy offset that corresponds to the change in the local kinetic energy of the electrons near the nucleus.

Later, this argument was used to explain the intensity of energetic electrons ejected in the forward direction when swift, partially stripped, heavy ions transverse a thin target [30]. From the reference frame in which these heavy ions are at rest, the target electrons appear as fast-moving projectiles and the energetic electrons appearing in the forward direction are due to close collisions between the nucleus and these electrons (hence the name binary encounter peak). The electron emission at zero degree for projectiles carrying bound electrons ("dressed ions") is much greater than that for bare ions. This enhancement is also attributed to screening and the related reduction in velocity of the electrons at very close proximity to the nucleus.

Our experiment is thus very similar to the one described by Schultz and Olson [30], but the laboratory frame now coincides with the frame in which the atom is at rest. We again see an enhancement of the intensity of electrons scattered over large angles.

The enhancement of the elastic cross section with screening is quantitatively well reproduced by quantum mechanical calculations such as the ELSEPA package from Salvat et al. [38] (Dirac partial-wave calculation of elastic scattering of electrons and positrons by atoms, positive ions, and molecules). The calculations also show the opposite effect in the case of high-energy positron scattering, namely, an elastic cross section smaller than the one given by the Rutherford formula. At high energies the Rutherford cross section, which depends on $Z^{2}$ only, lies in between the cross sections for electrons and positrons. This difference, roughly proportional to $Z^{3}$ at high energies, can be directly related to the Barkas effect in stopping power. In fact, it follows from Eq. (2) that the Barkas factor for the stopping power $\left(\mathrm{R}_{\text {Barkas }}\right)$, which quantifies the Barkas effect at close collisions, is determined by the transport cross section of the electron $\left[\sigma_{\mathrm{tr}}\left(e^{-}\right)\right]$and the transport cross section of the positron $\left[\sigma_{\mathrm{tr}}\left(e^{+}\right)\right]$in the reference frame where the ion is at rest according to $[24,25]$

$$
R_{\mathrm{Barkas}}=\frac{\sigma_{\mathrm{tr}}\left(e^{-}\right)-\sigma_{\mathrm{tr}}\left(e^{+}\right)}{\sigma_{\mathrm{tr}}\left(e^{-}\right)+\sigma_{\mathrm{tr}}\left(e^{+}\right)} .
$$

The DCS is determined by the electron-ion potential $V$ which can be written as [17]

$$
V=-\frac{(Z-q)}{r} \Phi(r)-\frac{q}{r} \exp \left(-r / a_{\mathrm{dyn}}\right),
$$

where $Z$ and $q$ are the atom (or ion) nuclear-charge and chargestate, $\Phi(r)$ is the screening function due to the bound electrons, and $a_{\mathrm{dyn}}$ is the screening length due to dynamical screening (about $v / \omega$, where $\omega$ is the plasmon frequency and $v$ is the ion velocity) [17,21].

For neutral atoms or slightly charged ions (the cations in a chemical compound $0 \leqslant q \lesssim 6$ ) the similarity between the Barkas effect and the strong enhancement (reduction) of the elastic cross section in comparison to the Rutherford one observed in high-energy electron (positron) scattering is evident. The interaction potential $V$ for a neutral, moving atom and for a stationary atom should be nearly the same as far as high-energy electrons are concerned. In fact, the origin of the Barkas effect at close collisions is the different influence of screening on the DCS for positively and negatively charged particles, which can be measured directly using either an electron or a positron as a projectile.

\section{ELECTRON RUTHERFORD BACKSCATTERING}

\section{A. Experimental procedure}

The experimental setup is thoroughly described in Refs. [42,43]. Two different spectrometers were employed, one for gas-phase measurements with $E_{0}$ between $600 \mathrm{eV}$ and $6 \mathrm{keV}$ and one for surface studies using energies up to $40 \mathrm{keV}$. In short, an electron beam with a small thermal spread is obtained using an electron gun with a $\mathrm{BaO}$ cathode. Slit lenses are used to focus and decelerate the electron beam from the scattering energy to the pass energy of (nominal) $200 \mathrm{eV}$. A 0.2-mm-wide conical slit aperture, placed $130 \mathrm{~mm}$ away from the sample, determines which electrons enter the analyzer. This means that the scattering angle is well determined (uncertainty about $0.1^{\circ}$ ). The energy resolution of the system is close to $0.3 \mathrm{eV}$ full width at half maximum.

\section{B. ERBS results}

In Fig. 1 we show ERBS spectra for four oxides $\left(\mathrm{Ta}_{2} \mathrm{O}_{5}\right.$, $\mathrm{HfO}_{2}, \mathrm{Nb}_{2} \mathrm{O}_{5}$, and $\mathrm{SiO}_{2}$ ) for an incident energy of $E_{0}=$ $40 \mathrm{keV}$ taken at normal incidence and a scattering angle of $135.5^{\circ}$. The corresponding elastic peaks for the $\mathrm{Ta}, \mathrm{Hf}, \mathrm{Nb}, \mathrm{Si}$, and $\mathrm{O}$ atoms are shown. Indeed, as seen in Fig. 1, each ERBS spectrum consists of two peaks corresponding to the elements present in the oxide. The huge elastic cross-section difference between the heavier elements and oxygen is reflected in their peak heights. From the ratio of the elastic-peak areas we obtain the ratio of the elastic cross sections taking into account the nominal stoichiometry of each oxide. This analysis is complicated by the presence of a background under the small oxygen peak. This is due to electrons scattered from the cation that have created also an electronic excitation. The oxygen peak is at a known energy loss and has a width that is almost the same for all oxides [33]. The area of the peak is determined such that after subtracting the $\mathrm{O}$ peak from the measured spectrum one obtains a background [red (solid) curve] that resembles the loss spectrum as is measured for much lower $E_{0}$ values $(5 \mathrm{keV})$ where the $\mathrm{O}$ and cation peak virtually coincide [44]. It is worthwhile to point out that the area ratio does not depend on the IMFP since this cancels out when taking the ratio of intensities for homogeneous thick materials. All films are thick enough so we do not need to consider the signal of the substrate $[36,44]$.

The ratio of cross sections for each element relative to oxygen is shown in Fig. 2 for $40 \mathrm{keV}$ and a scattering angle of $135.5^{\circ}$. This energy would correspond to projectiles with about $70 \mathrm{MeV} / \mathrm{u}$. The ERBS results were taken from Fig. 1 and the error bars represent the uncertainty in the background subtraction procedure. Note that for oxygen the ratio is one by definition. The curves correspond to theoretical calculations using the ELSEPA program [38] using default 


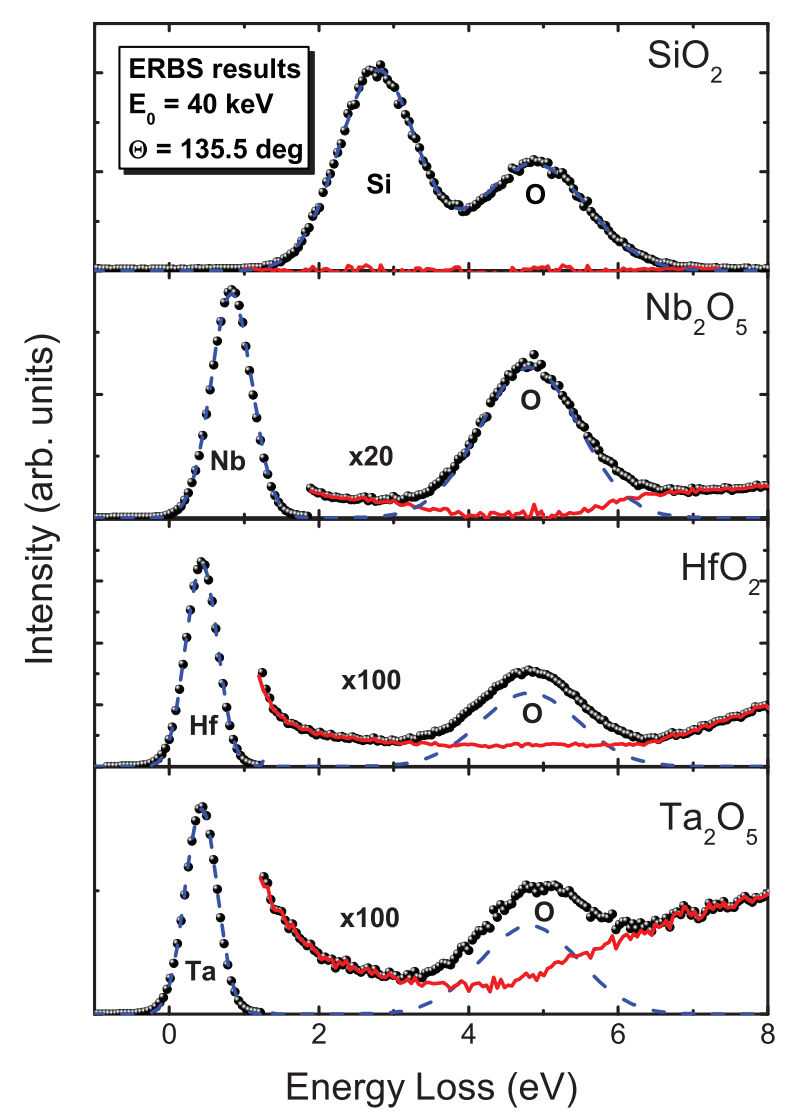

FIG. 1. (Color online) Spectra of $40 \mathrm{keV}$ electrons backscattered over $135.5^{\circ}$ from thick layers of $\mathrm{SiO}_{2}, \mathrm{Nb}_{2} \mathrm{O}_{5}, \mathrm{HfO}_{2}$, and $\mathrm{Ta}_{2} \mathrm{O}_{5}$. For all samples, the peaks of the heavy and light elements are well resolved but the measurement is not always completely background free. The $\mathrm{O}$ elastic peak was subtracted, with an area chosen such that the resulting background [red (solid) line] is similar in shape to the energy loss spectrum observed for $E_{0}=5 \mathrm{keV}$ (not shown here).

options (in particular, no absorption is considered) and, in the case of electrons, the results are the same as in the NIST electron elastic-scattering cross-section database [45]. As can be observed from this figure the calculations from ELSEPA agree reasonably well with the ERBS results and the calculated cross section ratios are larger than the simple estimate for the ratio $(Z / 8)^{2}$ from the Rutherford formula. This shows that at a large scattering angles the screening effect enhances the cross section for high values of $Z$ (as for Hf and Ta). ELSEPA calculations for positrons are also displayed in Fig. 2 and their cross sections are smaller than the Rutherford prediction. However, the screening effect for positrons is smaller than for electrons and indicates that the large enhancement observed for electrons on Ta or Hf is not totally due to the Barkas effect.

The ERBS technique can be also used at much lower energies to quantify hydrogen atoms. Figure 3 shows ERBS results for $2 \mathrm{keV}$ electrons impinging on water ice and vapor (a) and methane gas (b) with the same scattering angle as in Fig. 1. The elastic peaks of $\mathrm{O}$ and $\mathrm{H}$ from Fig. 3(a) [C and $\mathrm{H}$ from Fig. 3(b)] are well resolved. From the ratio of the areas of the elastic peaks and the number of $\mathrm{H}$ atoms in each molecule the ratio of the $\mathrm{O}(\mathrm{C})$ cross sections relative to $\mathrm{H}$ was determined for different electron energies as shown in Figs. 3(c) and 3(d). The error bars are mainly due to uncertainties in the background

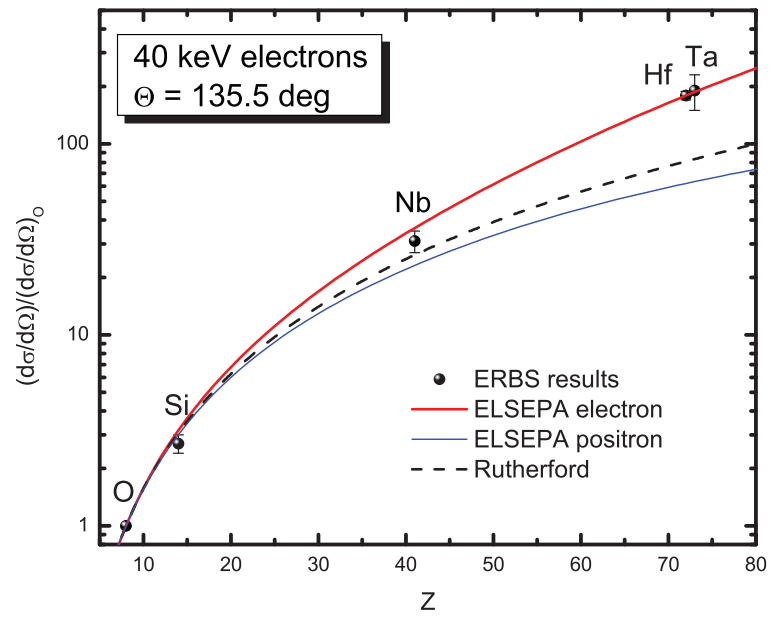

FIG. 2. (Color online) Experimental and theoretical values for the ratio of the elastic cross sections relative to $\mathrm{O}$ as a function of $Z$ for $40 \mathrm{keV}$ electrons and a scattering angle of $135.5^{\circ}$. The experimental values for $\mathrm{Si}, \mathrm{Nb}, \mathrm{Hf}$, and Ta were obtained from Fig. 1 after taking into account the nominal stoichiometry and background subtraction. The solid curves correspond to ELSEPA calculations for electrons (thick red line) and positrons (thin blue line). The dashed line (Rutherford) is a function $(Z / 8)^{2}$. For $\mathrm{O}$ the ratio is one by definition.

subtraction. The experimental ratio of intensities multiplied by the number of $\mathrm{H}$ atoms is compared to ELSEPA calculations for the cross-section ratio and show the same tendency as the results for heavy elements at much higher energies. The screening effect amounts to about $25 \%$ for $\mathrm{C}$ at $E_{0}=800 \mathrm{eV}$ (which corresponds to $1.5 \mathrm{MeV} / \mathrm{u}$ for $\mathrm{O}$ and $\mathrm{C}$ in the reference frame where the electrons are at rest). In contrast to the cases in Fig. 2, the screening effect is larger for positrons, and the average curve between the results for electrons and positrons lies below the Rutherford curve. This indicates a somewhat larger Barkas effect for strongly screened $1.5 \mathrm{MeV} / \mathrm{u} \mathrm{O}$ and $\mathrm{C}$ projectiles at small $e^{-}$-atom impact parameters of about $40 \%$. However, for ion stopping measurements there is no impact parameter selection, so the overall Barkas effect, calculated in the next section, is expected to be smaller.

The enhancement of the Ta and Hf peak seen in the ERBS spectra of oxides is in-line with the observation of an enhancement in the binary peak of the electron spectra near $0^{\circ}$ for high-energy ion experiments [29-31]. Most of these experiments are done at considerably lower energy (typically $1 \mathrm{MeV} / \mathrm{u}$ ) compared to the $73 \mathrm{MeV} / \mathrm{u}$ that is the equivalent ion kinetic energy in the $40 \mathrm{keV} e^{-}$scattering experiments described here. Around $40 \mathrm{keV}$ the elastic scattering cross section is a smooth function of angle, but at lower energies the situation changes for high $Z$ elements: quantum interference effects then cause sharp minima in the DCS. There are two reasons for using the high energy of $40 \mathrm{keV}$ : it is required for a clear separation of the elements involved but it also means that the Barkas effect can be studied without the competition of quantum interference effects. We can obtain element separation at much lower energies if we use protons as a reference element rather than oxygen. The low cross section of $\mathrm{H}$ requires that when studying high- $Z$ elements the number of $\mathrm{H}$ atoms present exceeds the heavy atom concentration by 

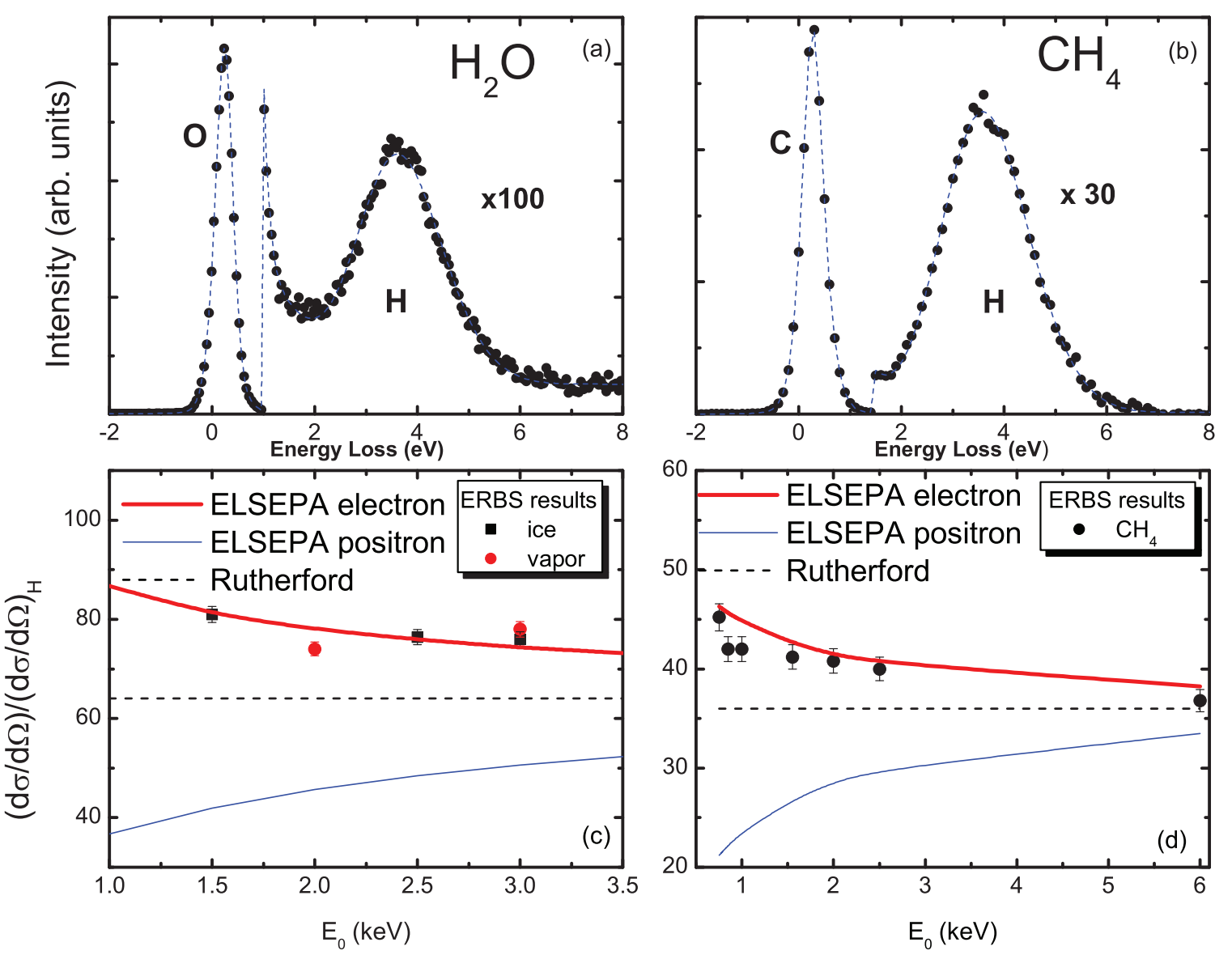

FIG. 3. (Color online) Upper panels: Measurements of $2 \mathrm{keV}$ electrons with normal incidence backscattered at $135^{\circ}$ from $\mathrm{H}_{2} \mathrm{O}$ and $\mathrm{CH}_{4}$ molecules. For a better visualization the $\mathrm{H}$ peak was multiplied by 100 in panel (a) and by 30 in panel (b). Lower panels: The ratio of cross sections relative to $\mathrm{H}$ as a function of the electron energy. ELSEPA calculations are also present for electrons (thick red line) and positrons (thin blue line) as well as the ratio obtained from the Rutherford cross section (dashed line).

at least an order of magnitude. As no suitable compounds exist such a study is best done using a gas-mixture of suitable concentrations in a cross beam configuration. By changing the electron beam energy or scattering angle we can monitor the quantum interference effects.

We illustrate this for Xe. The elastic DCS of electron scattering from $\mathrm{H}$ and $\mathrm{Xe}$, as obtained from ELSEPA are plotted in the top panels of Fig. 4. The $\mathrm{H}$ cross section is smooth and always close to Rutherford. The Xe cross section is still a smooth function at $10 \mathrm{keV}$ (but deviates from Rutherford mainly as a consequence of the Barkas effect) but this changes gradually below $2 \mathrm{keV}$ when sharp minima in the calculated elastic cross section develop. Indeed the dip in the Xe DCS causes dramatic variations in the $\mathrm{H}$-Xe peak intensity ratio. The sharp Xe peak at almost zero energy loss is followed by a much broader $\mathrm{H}$ peak at several eV energy loss. At $1.55 \mathrm{keV}$ the $\mathrm{Xe}$ peak area is $7.1 \times$ the $\mathrm{H}$ peak area, but at $750 \mathrm{eV}$ the $\mathrm{Xe}$ area is only $0.05 \times$ the $\mathrm{H}$ area. Such a change by a factor of 140 is in good agreement with the expectations based on the ELSEPA calculation. See Refs. [46,47] for more details about these gas-phase measurements.

In the ion-frame experiments measurements at the emission angle corresponding to the minimum in the DCS result in a splitting of the binary encounter peak [48]. The binary peak contains a Compton profile of the electron motion. The motion of the electron before the collision affects the effective scattering angle. Thus, under these conditions the intensity at the center of the peak is strongly reduced by the sharp minimum in the DCS, whereas the wings of the Compton profile (corresponding to slightly different scattering angles) are not. This causes the apparent split of the binary encounter peak.

We have demonstrated that phenomena playing an important role in ion stopping can also be studied in the frame where the ions are at rest. Now we proceed by using the theory developed to describe these electron-scattering experiments to study the Barkas effect in ion stopping.

\section{TRANSPORT CROSS-SECTION CALCULATIONS}

The theoretical calculations shown in Figs. 2 and 3 were performed at a specific scattering angle, namely $135.5^{\circ}$, for which the high-energy electron-scattering experiments were performed. For the evaluation of the Barkas factor related to the stopping power of ions in matter Eq. (4) we have to evaluate the transport cross section $\sigma_{\mathrm{tr}}$ which depends on all scattering angles. Formally this should be done in the center-of-mass frame but this is well approximated by the frame in which the atom is at rest. Using the default options for the ELSEPA 

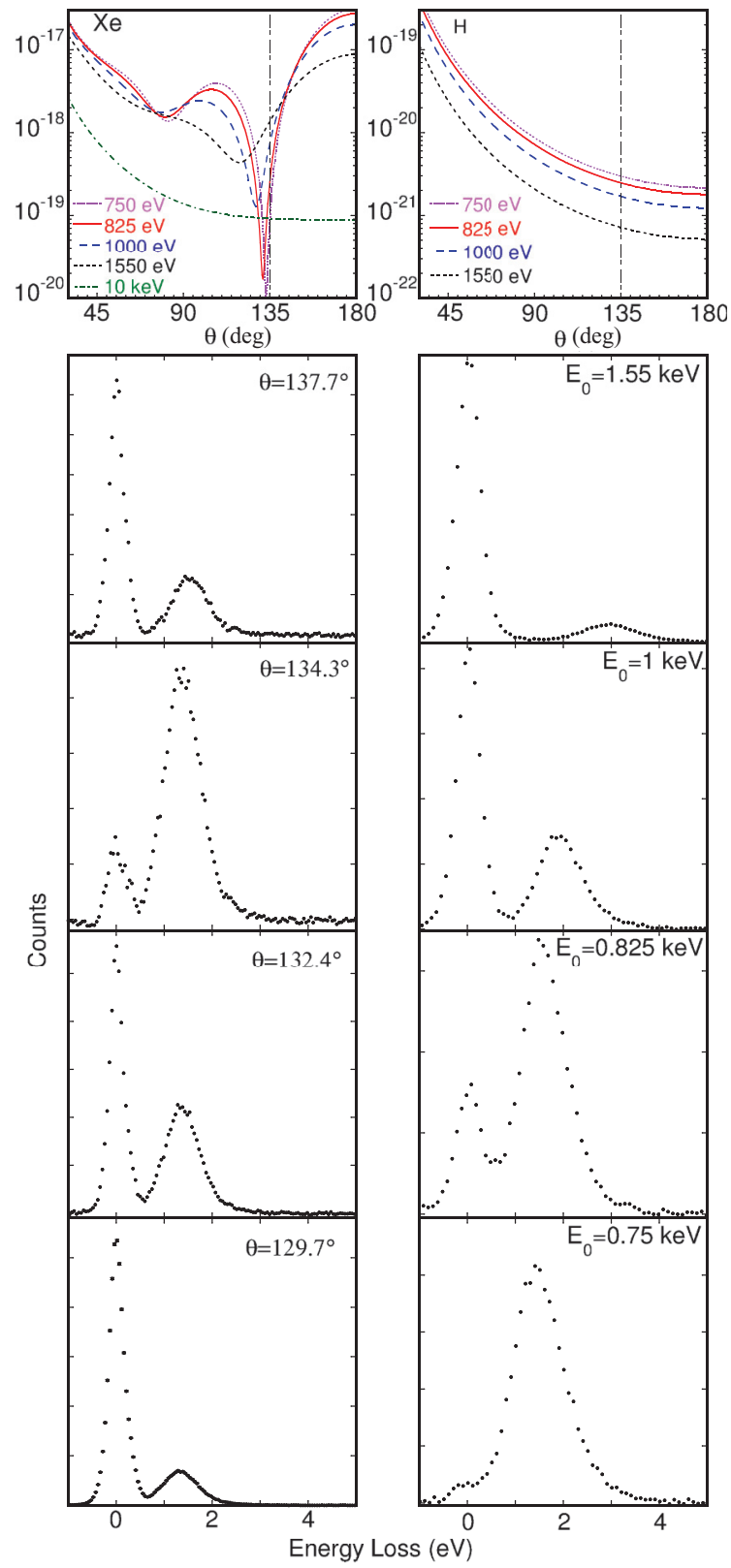

FIG. 4. (Color online) The top panels shows the calculated DCS for $\mathrm{Xe}$ and $\mathrm{H}$ atoms (in a.u.) at the energies indicated. The four panels at the left show the elastic peak of a Xe- $\mathrm{H}_{2}$ mixture at $750 \mathrm{eV}$ at the angles as indicated. The right panels show the elastic peak of a (different) $\mathrm{Xe}-\mathrm{H}_{2}$ mixture taken at $135^{\circ}$ and at the energies as indicated.

program (in particular, without considering absorption), we calculated $\sigma_{\text {tr }}$ of electrons and positrons for all elements and several energies ranging from 0.1 to $40 \mathrm{keV}$ for neutral ions $(q=0)$ and the screening function $\Phi(r)$ given by Hartree-Fock (HF) densities as detailed in Ref. [38]. From these transport cross sections we obtain the Barkas factor $\left(R_{\text {Barkas }}\right)$ according to Eq. (4). Figure 5 shows the results as a function of the dimensionless parameter $\eta$ defined as

$$
\eta=\frac{Z / \xi}{E_{0}}=\frac{2 Z}{\xi v^{2}}
$$

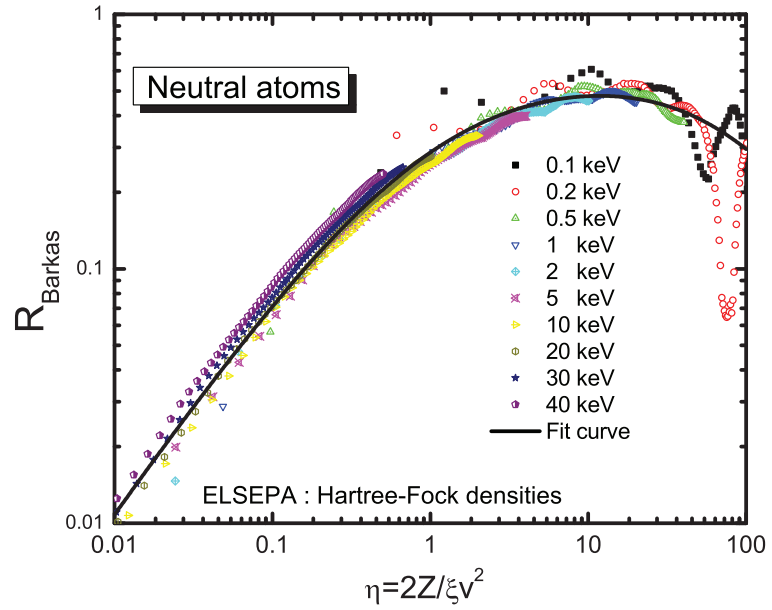

FIG. 5. (Color online) Barkas factor according to Eq. (4) as a function of the dimensionless parameter defined in Eq. (6). The calculations were done using the ELSEPA code for all elements and using HF densities for neutral atoms. The energies indicated are the kinetic energies of electrons scattering from a stationary nucleus; $1 \mathrm{keV}$ corresponds to $1.836 \mathrm{MeV} / \mathrm{u}$ in an ion beam experiment.

where $\xi$ is a screening length. The $Z / \xi$ term is a constant, which has a dimension of energy, and can be obtained, as described by Lindhard [10], from the expansion of the potential for small distances $r$, where the screened interaction potential can be described as a Coulomb potential shifted uniformly by $Z / \xi$. Thus the scattering near the nucleus is described as scattering from a bare Coulomb potential, but with the kinetic energy of the scattered particle changed by $Z / \xi$ (corresponding to an decrease for electrons and an increase for positrons). $\eta$ is this change normalized by the incoming kinetic energy.

For $\xi$ we used the screening length from the Brandt-Kitagawa (BK) statistical model [49], namely $\xi=$ $\Lambda_{\mathrm{BK}}(Z, q=0)$, where

$$
\Lambda_{\mathrm{BK}}(Z, q)=\frac{0.48(1-q / Z)^{2 / 3}}{Z^{1 / 3}[1-1 / 7(1-q / Z)]} .
$$

This gives (in $\mathrm{eV}$ ) an offset energy $Z / \xi$ of $\approx 48.6 Z^{4 / 3}$ for $q=0$. First we compare our estimate of the Barkas factor based on ELSEPA with recent calculations based on classical mechanics [25]. For $\eta<1$ the Barkas factor, when plotted as a function of the dimensionless parameter $\eta$, falls on a single curve as in the classical calculation of Ref. [25]. However, for large values of $\eta$, the scaling breaks down in the present calculation because of quantum effects. As a matter of fact local minima are observed in the differential cross section for particular scattering angles for energies smaller than $\approx 2 \mathrm{keV}$ and large nuclear charge $Z$ [38]. For the transport cross section, oscillations are also found as a function of $Z$ [22] due to interferences and shell effects. Nevertheless, for low values of $\eta$ (in the so-called perturbative regime), a single universal curve can be obtained. A best fit to calculations is given by

$$
R_{\mathrm{Barkas}}=\frac{a \eta}{1+b \eta^{0.25}+c \eta+d \eta^{2}},
$$


TABLE I. Values obtained for the constants in the "universal curve" given in Eq. (8) for neutral atoms and charged ions using either a Hartree-Fock (HF) derived electron density or the density of the Brandt-Kitagawa (BK) model for neutral atoms and ions.

\begin{tabular}{lcclll}
\hline \hline Model & $a$ & $b$ & \multicolumn{1}{c}{$c$} & \multicolumn{1}{c}{$d$} \\
\hline HF (neutral) & 2.1 & 2.8 & 3.4 & 0.035 \\
BK (neutral) & 0.9 & 0 & 1.4 & 0.04 \\
BK (ion) & 0.9 & 0 & $1.4(Z / N)^{1.5}$ & $0.04(Z / N)^{2.5}$ \\
\hline \hline
\end{tabular}

with values of $a, b, c$, and $d$ as given in Table I. Similar curves were obtained in the framework of classical mechanics in Ref. [25] for neutral ions and in Refs. [24,25] for bare ions, where the screening length $\xi$ should be replaced by a value proportional to the adiabatic radius $v / \omega$.

Now we consider the Barkas factor as a function of the ion charge state. In order to generate the curves displayed in Fig. 6 we used the interaction potential from Eq. (5) with the BK screening function $\Phi(r)=\exp \left[-r / \Lambda_{\mathrm{BK}}(Z, q)\right]$ that depends on $Z$ and $q$ according Eq. (7). In addition we considered $a_{\mathrm{dyn}}=$ $v / \omega$, with $\omega=16 \mathrm{eV}$. The charge density corresponding to the BK screening function was used as input for the ELSEPA code. In addition we turned off the exchange for this calculation. The calculations were performed for different energies (from 0.5 to $5 \mathrm{keV}$ ) and plotted using the average screening length $\xi$ defined as

$$
\frac{Z}{\xi}=\frac{Z-q}{\Lambda_{\mathrm{BK}}(Z, q)}+\frac{q}{a_{\mathrm{dyn}}} .
$$

This expression is based on the small $r$ expansion of Eq. (5).

From Fig. 6 we find that for $q<Z / 2$ the Barkas factors obtained for different energies still fall on a single curve when plotted as a function of $\eta$, but the curves for different charge states do not coincide, and the magnitude of the Barkas factor decreases with $q$. Further inspection of this figure shows that $R_{\text {Barkas }}$ values for different projectile charge states all merge with the curve for neutral atoms at high velocities (small $\eta$ values). Here the dynamical screening is less important compared to the screening due to bound electrons. The breakdown of the scaling for different projectile charge states at larger $\eta$ values is due to the interplay between the two screening functions contributing to Eq. (5), each with a different characteristic length.

For the limiting cases of a neutral atom and a fully stripped atom there is only a single screening length resulting in a simple Yukawa type potential and hence the graphs for $q=0$ and $q=Z$ are identical in Fig. 6, but the respective values of $\xi$ are totally different. Thus there is a single universal curve for the Barkas factor (here for $\eta<1$ ) and in classical calculations (for all $\eta$ values) [25] for the neutral atom and the fully stripped ions, but for partially stripped ions the curves are $q$ dependent. For partially stripped ions there are two Yukawa-type screening

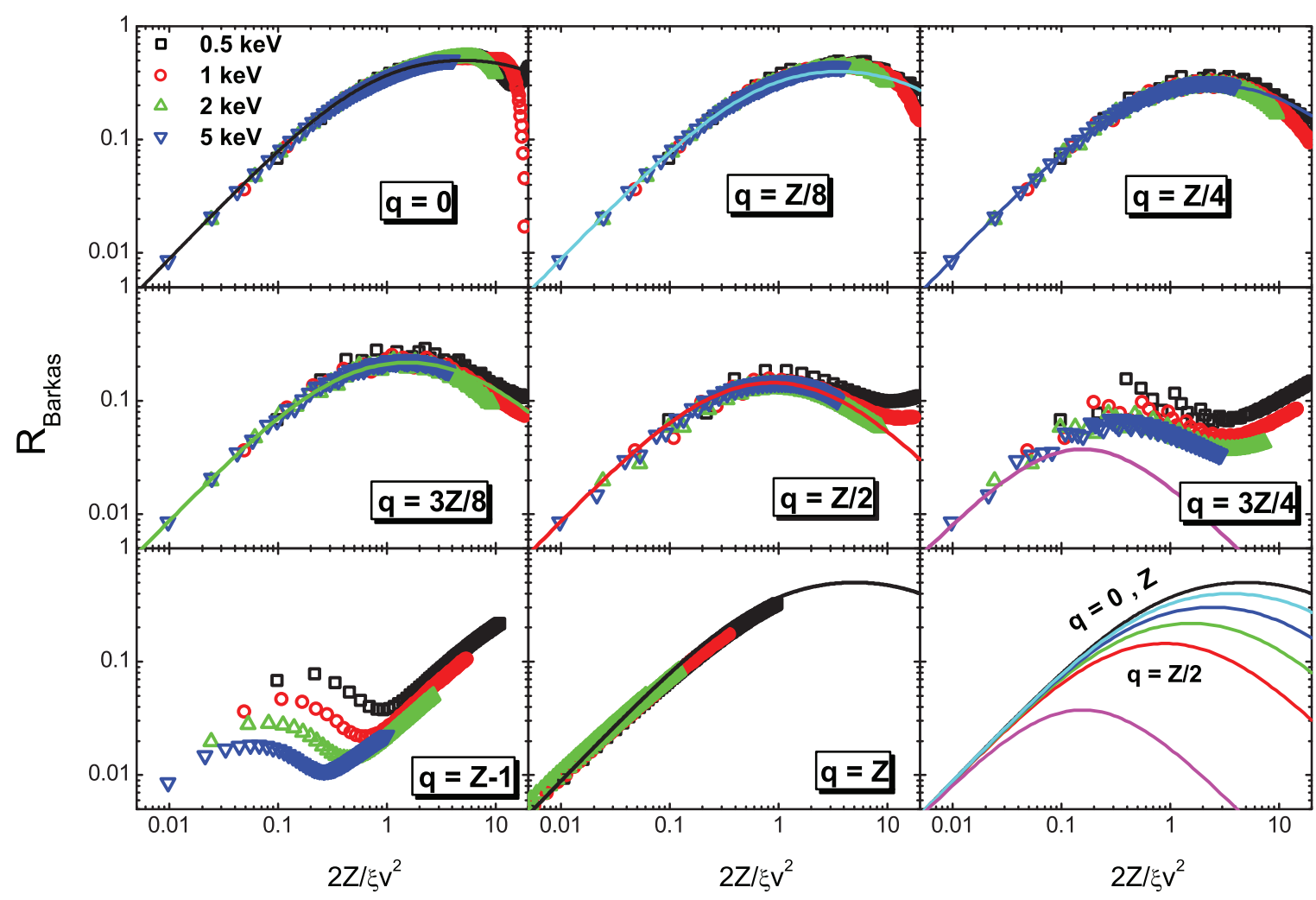

FIG. 6. (Color online) Barkas factor as a function of the dimensionless parameter defined in Eq. (6) using the average screening length from Eq. (9). The calculations were done using the ELSEPA code for all elements and for projectile charge states as indicated using the atomic potential according to the BK model. The net charge of the ion is screened using $a_{\mathrm{dyn}}=v / \omega$ with $\omega=16 \mathrm{eV}$. This approach is successful in mapping $R_{\mathrm{Barkas}}$ for different energies on a single curve for charge states up to $q=Z / 2$. The last panel shows the fit based on Eq. (8) for charge states up to $q=3 Z / 4$ and the fully stripped ion. 
functions: one describing screening by bound electrons and one the dynamical screening. The scaling proposed in Eq. (9), which combines both screening types, describes the Barkas factor for different $E_{0}$ values quite well as long as the charge of the ion is less than $Z / 2$.

Only for a fully stripped ion is $\eta$ proportional to $Z$. This is the behavior expected for the $Z^{3}$ contribution to the stopping power, which results in a Barkas effect proportional to $Z$. For neutral atoms and partial stripped ions these calculations indicate a more complicated dependence of the Barkas effect on $Z$.

\section{CONCLUSIONS}

In this work we explore the connection between the Barkas effect in the stopping power of ions and the screeninginduced enhancement of the differential cross section in highenergy electron-scattering experiments. ERBS measurements at $40 \mathrm{keV}$ for $\mathrm{Ta}_{2} \mathrm{O}_{5}$ and $\mathrm{HfO}_{2}$ show a large screening-induced enhancement(a factor of $\approx 2$ relative to Rutherford) for Ta and Hf. For water and methane at 0.75 to $6 \mathrm{keV}$ the enhancement for $\mathrm{O}$ and $\mathrm{C}$ is $\approx 1.25$. The results are in agreement with calculations of the elastic cross section from the ELSEPA program [38]. In combination with ELSEPA results for $\mathrm{keV}$ positron scattering we can extract the Barkas factor in the ion stopping power at $\mathrm{MeV} / \mathrm{u}$ energies. At $70 \mathrm{MeV} / \mathrm{u}$ ion energy (corresponding to an electron-scattering energy of $40 \mathrm{keV}$ ) the Barkas effect is about $50 \%$ for (nearly) neutral Ta and Hf ions. A similar value was found for $1.5 \mathrm{MeV} / \mathrm{u} \mathrm{O}$ and $\mathrm{C}$ (nearly) neutral ions. For ions with a substantial charge the Barkas effect at close collisions is smaller.

The ELSEPA code was also used to investigate if it is possible to describe approximately the size of the Barkas effect for a range of different conditions with a simple formula. For this purpose the Barkas factor was calculated for all elements at many different energies. A universal curve was found for (nearly) neutral projectiles based on a dimensionless parameter $\eta$ except for large $Z$ and low energies, where quantum interference effects are important. For ions with a large charge the Barkas effect is smaller than that derived from the curve for (nearly) neutral ions, but up to $q=Z / 2$ the Barkas factor can be described quite well by a simple formula.

\section{ACKNOWLEDGMENTS}

One of the authors (P.L.G) acknowledges the Brazilian agency CAPES (Grant No. 102209/12-3) for financial support. The authors want to thank Dr. D. K. Venkatachalam and S. K. Nandi for the oxide samples, and Professor Weigold for critically reading the manuscript. This work is made possible by a grant from the Australian Research Council.
[1] H. Bethe, Ann. Phys. 397, 325 (1930).

[2] H. H. Andersen, J. F. Bak, H. Knudsen, and B. R. Nielsen, Phys. Rev. A 16, 1929 (1977).

[3] L. H. Andersen, P. Hvelplund, H. Knudsen, S. P. Möller, J. O. P. Pedersen, E. Uggerhöj, K. Elsener, and E. Morenzoni, Phys. Rev. Lett. 62, 1731 (1989).

[4] A. Adamo et al., Phys. Rev. A 47, 4517 (1993).

[5] S. P. Møller, E. Uggerhøj, H. Bluhme, H. Knudsen, U. Mikkelsen, K. Paludan, and E. Morenzoni, Phys. Rev. A 56, 2930 (1997).

[6] F. M. Smith, W. Birnbaum, and W. H. Barkas, Phys. Rev. 91, 765 (1953).

[7] W. H. Barkas, W. Birnbaum, and F. M. Smith, Phys. Rev. 101, 778 (1956).

[8] W. H. Barkas, J. N. Dyer, and H. H. Heckman, Phys. Rev. Lett. 11, 26 (1963).

[9] J. C. Ashley, R. H. Ritchie, and W. Brandt, Phys. Rev. B 5, 2393 (1972).

[10] J. Lindhard, Nucl. Instrum. Methods 132, 1 (1976).

[11] G. Schiwietz, Phys. Rev. A 42, 296 (1990).

[12] G. Schiwietz, U. Wille, R. D. Muiño, P. D. Fainstein, and P. L. Grande, J. Phys. B 29, 307 (1996).

[13] P. L. Grande and G. Schiwietz, in Advances in Quantum Chemistry, edited by J. R. Sabin, E. Brändas, and R. CabreraTrujillo (Elsevier, Academic Press, San Diego, 2004), Vol. 45.

[14] P. L. Grande and G. Schiwietz, Phys. Rev. A 47, 1119 (1993).

[15] F. Grüner and F. Bell, Nucl. Instrum. Methods Phys. Res., Sect. B 245, 15 (2006).
[16] J. M. Pitarke, R. H. Ritchie, and P. M. Echenique, Phys. Rev. B 52, 13883 (1995).

[17] P. Sigmund, Stopping of Heavy Ions-A Theoretical Approach, 1st ed. (Springer, Berlin, Heidelberg, New York, 2004).

[18] P. Sigmund and A. Schinner, Nucl. Instrum. Methods Phys. Res., Sect. B 195, 64 (2002).

[19] P. Sigmund and A. Schinner, Eur. Phys. J. D. 12, 425 (2000).

[20] P. Sigmund and A. Schinner, Nucl. Instrum. Methods Phys. Res., Sect. B 212, 110 (2003).

[21] A. F. Lifschitz and N. R. Arista, Phys. Rev. A 57, 200 (1998).

[22] N. R. Arista, Nucl. Instrum. Methods Phys. Res., Sect. B 195, 91 (2002).

[23] P. Grande and G. Schiwietz, CasP program, http://www.casp-program.org/.

[24] N. R. Arista, P. L. Grande, and A. F. Lifschitz, Phys. Rev. A 70, 042902 (2004).

[25] C. Archubi, I. Abril, R. Garcia-Molina, and N. R. Arista, Nucl. Instrum. Methods Phys. Res., Sect. B 316, 88 (2013).

[26] A. Sørensen, Nucl. Instrum. Methods Phys. Res., Sect. B 48, 10 (1990).

[27] G. Maynard, G. Zwicknagel, C. Deutsch, and K. Katsonic, Phys. Rev. A 63, 052903 (2001).

[28] J. E. Miraglia, Phys. Rev. A 68, 022904 (2003).

[29] C. O. Reinhold, D. R. Schultz, and R. E. Olson, J. Phys. B 23, L591 (1990).

[30] D. R. Schultz and R. E. Olson, J. Phys. B 24, 3409 (1991).

[31] M. Sataka, M. Imai, Y. Yamazaki, K. Komaki, K. Kawatsura, Y. Kanai, H. Tawara, D. R. Schultz, and C. O. Reinhold, J. Phys. B 27, L171 (1994). 
[32] M. Vos and M. Went, J. Electron Spectrosc. Relat. Phenom. 155, 39 (2007).

[33] M. Went and M. Vos, Nucl. Instrum. Methods Phys. Res., Sect. B 266, 998 (2008).

[34] M. Vos, R. McEachran, E. Weigold, and R. Bonham, Nucl. Instrum. Methods Phys. Res., Sect. B 300, 62 (2013).

[35] M. Vos, E. Weigold, and R. Moreh, J. Chem. Phys. 138, 044307 (2013).

[36] P. L. Grande, M. Vos, D. K. Venkatachalam, S. K. Nandi, and R. G. Elliman, Appl. Phys. Lett. 103, 071911 (2013).

[37] J. R. Tesmer and M. A. Nastasi, Handbook of Modern Ion Beam Materials Analysis: Materials Research Society, 1st ed. (MRS, Pittsburgh, 1995).

[38] F. Salvat, A. Jablonski, and C. J. Powell, Comput. Phys. Commun. 165, 157 (2005).

[39] A. Jablonski, F. Salvat, and C. J. Powell, Surf. Interface Anal. 37, 1115 (2005).

[40] G. I. Watson, J. Phys.: Condens. Matter 8, 5955 (1996).
[41] M. Vos, C. A. Chatzidimitriou-Dreismann, T. Abdul-Redah, and J. Mayers, Nucl. Instrum. Methods Phys. Res., Sect. B 227, 233 (2005).

[42] M. Went, M. Vos, and R. G. Elliman, J. Electron Spectrosc. Relat. Phenom. 156-158, 387 (2007).

[43] M. Vos, M. Went, G. Cooper, and C. ChatzidimitriouDreismann, J. Phys. B 41, 135204 (2008).

[44] M. Vos, P. L. Grande, S. K. Nandi, D. K. Venkatachalam, and R. G. Elliman, J. Appl. Phys. 114, 073508 (2013).

[45] C. J. Powell, A. Jablonski, and F. Salvat, Surf. Interface Anal. 37, 1068 (2005).

[46] M. Vos, J. Phys. B 43, 215201 (2010).

[47] R. P. McEachran and M. Vos, Phys. Rev. A 85, 032703 (2012).

[48] C. O. Reinhold, D. R. Schultz, R. E. Olson, C. Kelbch, R. Koch, and H. Schmidt-Böcking, Phys. Rev. Lett. 66, 1842 (1991).

[49] W. Brandt and M. Kitagawa, Phys. Rev. B 25, 5631 (1982). 